\title{
Article \\ Chemosensory Optode Array Based on Pluronic-Stabilized Microspheres for Differential Sensing
}

\author{
Aleksandra Kalinowska (D), Magdalena Wicik, Patrycja Matusiak and Patrycja Ciosek-Skibińska *D \\ Chair of Medical Biotechnology, Faculty of Chemistry, Warsaw University of Technology, Noakowskiego 3 , \\ 00-664 Warsaw, Poland; aleksandra.kalinowska3.dokt@pw.edu.pl (A.K.); mwicik@ch.pw.edu.pl (M.W.); \\ pmatusiak@ch.pw.edu.pl (P.M.) \\ * Correspondence: pciosek@ch.pw.edu.pl
}

check for updates

Citation: Kalinowska, A.; Wicik, M.; Matusiak, P.; Ciosek-Skibińska, P. Chemosensory Optode Array Based on Pluronic-Stabilized Microspheres for Differential Sensing. Chemosensors 2022, 10, 2. https://doi.org/10.3390/ chemosensors10010002

Academic Editor: Zhihong Liu

Received: 15 November 2021

Accepted: 20 December 2021

Published: 22 December 2021

Publisher's Note: MDPI stays neutral with regard to jurisdictional claims in published maps and institutional affiliations.

Copyright: (C) 2021 by the authors. Licensee MDPI, Basel, Switzerland. This article is an open access article distributed under the terms and conditions of the Creative Commons Attribution (CC BY) license (https:// creativecommons.org/licenses/by/ $4.0 /)$
Abstract: Differential sensing techniques are becoming nowadays an attractive alternative to classical selective recognition methods due to the "fingerprinting" possibility allowing identifying various analytes without the need to fabricate highly selective binding recognition sites. This work shows for the first time that surfactant-based ion-sensitive microspheres as optodes in the microscale can be designed as cross-sensitive materials; thus, they are perfect candidates as sensing elements for differential sensing. Four types of the newly developed chemosensory microspheres-anion- and cation-selective, sensitive toward amine- and hydroxyl moiety-exhibited a wide range of linear response (two to five orders of magnitude) in absorbance and/or fluorescence mode, great time stability (at least 2 months), as well as good fabrication repeatability. The array of four types of chemosensitive microspheres was capable of perfect pattern-based identification of eight neurotransmitters: dopamine, epinephrine, norepinephrine, $\gamma$-aminobutyric acid (GABA), acetylcholine, histamine, taurine, and phenylethylamine. Moreover, it allowed the quantification of neurotransmitters, also in mixtures. Its selectivity toward neurotransmitters was studied using $\alpha$ - and $\beta$-amino acids (Ala, Asp, Pro, Tyr, taurine) in simulated blood plasma solution. It was revealed that the chemosensory optode set could recognize subtle differences in the chemical structure based on the differential interaction of microspheres with various moieties present in the molecule. The presented method is simple, versatile, and convenient, and it could be adopted to various quantitative and qualitative analytical tasks due to the simple adjusting of microspheres components and measurement conditions.

Keywords: pattern-based sensing; differential sensing; fingerprinting; optodes; microspheres; neurotransmitters; sensor array

\section{Introduction}

The basis of any optical sensor is a molecule that changes optical properties in response to the presence of the analyte of interest [1]. There are many dyes for direct hydrogen ions $(\mathrm{pH})$, oxygen, or other important analytes sensing - in such cases, one molecule serves as both the receptor for the analyte and indicator that reports the analyte recognition event. For ion sensing, a variety of optical ion sensors have been discovered, such as fluorescent molecular probes, surface-modified nanomaterials, biologically expressed proteins, etc. Indirect sensing methods, where separate molecules are used for analyte recognition and signaling, allow for significant expansion of the range of detectable analytes. An indirect approach is very advantageous - sensor specificity is altered by only changing the analyte receptor, without the need for synthetizing new compounds with the reporter covalently attached to the binding moiety, which is essential in direct sensing routes [1-3].

Ionophore-based ion-selective optical sensors, known also as ion-selective optodes or ionophore-based optical sensors (IBOS), or simply optodes, take advantage of the excellent selectivity of a wide range of various commercially available ionophores (ion receptors), which are well known and often applied in potentiometric sensors (Ion-Selective Electrodes, ISEs). As the optodes rely on the indirect sensing strategy, the recognition part (an 
ionophore for ion recognition) and the optical reporter ( $\mathrm{pH}$ indicator-chromoionophore) are two separate molecules [2-5]. Therefore, optodes are usually formulated with a chromoionophore, ionophore, and a charge-balancing additive, which are all contained in a hydrophobic polymer matrix (an optodecocktail). In such a sensing system, the target ion binding to an ionophore is indirectly coupled to the protonation state of the $\mathrm{pH}$ indicator via maintaining charge balance in the hydrophobic phase. Therefore, the analyte activity can be detected indirectly by monitoring the hydrogen ion level in the sensor, which is reported by the change of optical properties of the chromoionophore. Such an indirect approach is very advantageous and versatile, since there is no need to synthetize the molecule having both a recognition part and reporter part covalently attached. Instead, various chromoionophores can be applied together with receptors that do not change optical properties in the act of binding the target analyte. Fluorescence intensity and absorbance are by far the two major readout modes for optodes. However, during the last few years, also other types of signals, such as fluorescence decay time, refractive index, or surface plasmon resonance, have also been successfully applied $[5,6]$.

The optodes have proven to be a robust and versatile tool for measuring various ion concentrations (sodium, calcium, potassium, nitrate, thiocyanate, etc.). Their performance can be tuned to obtain desired analytical properties by adjusting components of the optodecocktail. In the last years, a trend toward the advanced miniaturization of IBOS is observed. The optode components in the form of optodecocktail can be further processed to obtain chemosensitive microspheres, nanospheres, or micelles, in which surfactants are applied for emulsion stabilization [5-7]. When ion optodes are miniaturized from the bulk to the nanoscale out of the same material, no dramatic change in the selectivity pattern is expected. The reason for this assumption is that the selectivity for ion optodes mainly depends on the ionophores. Without incorporating ionophores, the selectivity, in general, follows the Hoffmeister series. By the careful designing composition of the cocktail, such nano/micro-optical sensors of differentiated selectivity can be developed. Moreover, such miniaturized sensors usually have much shorter response times and smaller required sample volume, which are an advantage of optical micro/nanospheres over ISEs and bulk optodes $[5,6,8]$.

So far, various microsphere-based optodes have been reported in the literature. They were successfully applied for the determination of different samples, including in trace analysis, and as biocompatible nanosensors toward in vitro and in vivo imaging applications [3,5]. However, so far, to our best knowledge, surfactant-stabilized microspheres have not been used for differential sensing $[1,9,10]$.

Therefore, in this work, we propose the application of the newly developed chemosensory microspheres for the identification of model bioanalytes-neurotransmitters, by differential sensing. First, the formulations of the optode cocktails were tested if they receive sensitivity toward various lipophilic ions and compounds with amino- and -OH groups. Time stability and fabrication repeatability were checked as important issues for sensor development. Then, the fluorimetric and spectrophotometric responses of optode microspheres obtained in the presence of neurotransmitters were examined to show the great potential of optode microspheres for differential sensing.

\section{Experimental}

\subsection{Reagents and Materials}

Dopamine hydrochloride, L-epinephrine, L-norepinephrine, $\gamma$-aminobutyric acid (GABA), acetylcholine chloride, histamine, taurine, phenylethylamine, sodium phosphate monohydrate, disodium phosphate dodecahydrate, Tris-HCl, Pluronic F127 were supplied by Sigma-Merck (Poznań, Poland). Milli-Q water was used for the preparation of all aqueous solutions, including phosphate buffer $\mathrm{pH} 7.4$, Tris- $\mathrm{HCl}$ buffer $\mathrm{pH} 9.0$, and simulated Blood Plasma Solution (BPS: $6.800 \mathrm{~g} / \mathrm{L} \mathrm{NaCl}, 0.200 \mathrm{~g} / \mathrm{L} \mathrm{CaCl}_{2}, 0.400 \mathrm{~g} / \mathrm{L} \mathrm{KCl}, 0.205 \mathrm{~g} / \mathrm{L}$ $\mathrm{MgSO}_{4} * 7 \mathrm{H}_{2} \mathrm{O}, 2.200 \mathrm{~g} / \mathrm{L} \mathrm{NaHCO}_{3}, 0.318 \mathrm{~g} / \mathrm{L} \mathrm{Na}_{2} \mathrm{HPO}_{4} * 12 \mathrm{H}_{2} \mathrm{O}, 0.026 \mathrm{~g} / \mathrm{L} \mathrm{NaH}_{2} \mathrm{PO}_{4}$ ) according to [11]. 
Plasticizers (2-Nitrophenyl octyl ether-o-NPOE, Bis(2-Ethylhexyl) sebacate-DOS, bis(2-Ethylhexyl)phthalate-DOP), lipophilic salts (Tridodecylmethylammonium chlorideTDMAC, Potassium tetrakis3,5-bis(trifluoromethyl)phenyl borate-KTFPB, sodium tetraphenylborate-NaTPB), Chromoionophore I (9-(Diethylamino)-5-(octadecanoylimino)5H-benzo[a]phenoxazine, ETH 5294), Chromoionophore IV (5-Octadecanoyloxy-2-(4nitrophenylazo)phenol, ETH 2412), Chromoionophore XI (Fluorescein octadecyl ester, ETH 7061), and ionophores were obtained from Fluka (Selectophore). Freshly distilled tetrahydrofuran (Fluka) was used as a solvent for the microspheres components. All chemicals were used as received.

\subsection{Preparation and Measurements of Optode Microspheres}

We prepared 4 types of optode microspheres: sensitive toward compounds with an amine group, sensitive toward compounds with an $-\mathrm{OH}$ group, with generic anion sensitivity and with generic cation sensitivity (Tables 1 and 2). Their formulations were based on literature data on optode membranes $[7,12,13]$, and our previous experience on lipophilic ion-sensitive membranes e.g., [14-16].

The optode microspheres were prepared from a mixture of chromoionophore $(0.5-0.9 \mathrm{mg})$, plasticizer (4.0 mg), surfactant Pluronic F127 (12.5 mg), and in the case of amine sensitive microspheres, also amine ionophore I $(2.0 \mathrm{mg})$. Lipophilic salts were added to the mixture in all cases except $-\mathrm{OH}$ sensitive microspheres: $0.6 \mathrm{mg}$ TDMAC in anion-selective microspheres, $1.6 \mathrm{mg}$ KTFPB in cation-selective microspheres, $1.0 \mathrm{mg}$ NaTPB in amineselective microspheres. The following chromoionophores were used: Chromoionophore XI in anion-selective and $-\mathrm{OH}$ sensitive microspheres, Chromoionophore I in cation-selective microspheres, Chromoionophore IV in amine-selective microspheres. Plasticizers (o-NPOE, DOS, and DOP) were applied to anion-selective, cation-selective, amine-selective as well as -OH-sensitive microspheres, respectively.

Each mixture was dissolved in $1.5 \mathrm{~mL}$ THF to form a homogenous solution, and then $0.5 \mathrm{~mL}$ portions were pipetted to $4.5 \mathrm{~mL}$ of deionized water on a vortex. The resulting clean mixture was blown using air pumped by a peristaltic pump to remove THF, and thus, clear particle suspension was obtained. Detailed preparation of microsphere suspensions was presented in [7]. For all experiments except the study of stability in time, freshly prepared optode microsphere suspensions were used. Their size in a range of a few micrometers was confirmed by confocal microscopy (the microscope images were provided in our recent publications $[17,18])$.

Spectrophotometric and spectrofluorimetric measurements were performed by a Synergy MX Multi-Mode Microplate Reader (BioTek Instruments, Inc., Winooski, VT, USA), using polystyrene Greiner CELLSTAR ${ }^{\circledR}$ 96-well microplates (Greiner Bio-One $\mathrm{GmbH}$, Kremsmünster, Austria). In case of calibrations as well as neurotransmitters identification, 96-well plates were prepared as follows: microsphere suspensions $(100 \mu \mathrm{L})$ were pipetted to each well, and then buffered solution of model analyte $(100 \mu \mathrm{L})$ in respective concentration or buffered solution of neurotransmitter $(100 \mu \mathrm{L}$ of $1 \mathrm{mM}$ solution) was added. Thus, the final concentration of neurotransmitters in wells was $500 \mu \mathrm{M}$ and they were measured in 8 replications. Phosphate buffer $\mathrm{pH} 7.4$ was applied to the measurements of anion-selective microspheres, whereas Tris- $\mathrm{HCl}$ buffer $\mathrm{pH} 9.0$ was used in all other cases. 
Table 1. Schematic representation of the fabricated microspheres, their composition and mechanisms of target analyte recognition with optical signal generation. The mechanisms are based on the extraction of the analyte from the water environment (aq subscript) to the lipophilic inner of the microsphere (m subscript). Each type of microsphere solution is based on plasticizer emulsion stabilized by non-ionic surfactant Pluronic, having hydrophobic and hydrophilic domains (PPO and PEO, respectively). Droplets of the plasticizer contain all components needed for the extraction of target analytes (lipophilic salt/ionophore) and signal generation (IND).
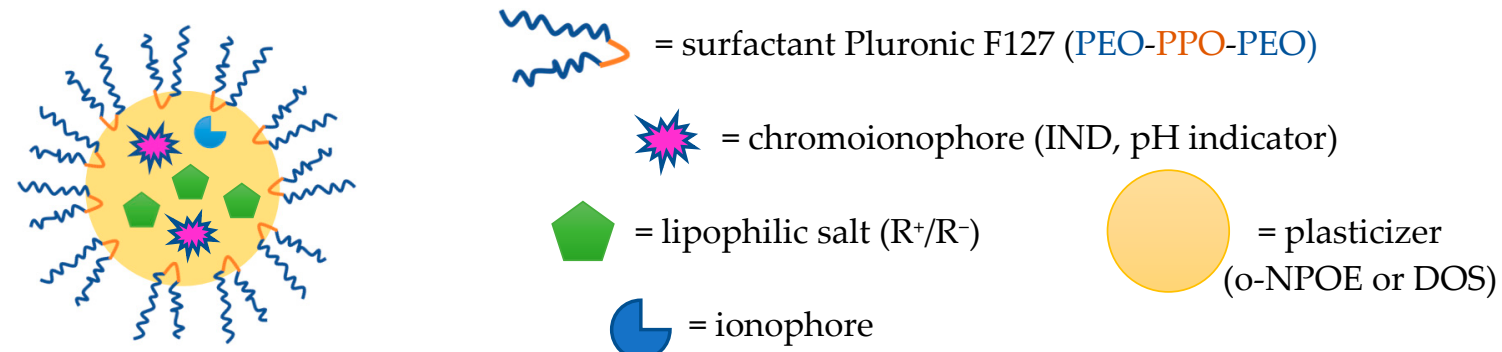

1

$$
\underbrace{\mathrm{IND}_{\mathrm{m}}+\mathrm{R}_{\mathrm{m}}^{+}+\mathrm{H}_{\mathrm{aq}}^{+}+\mathrm{AN}_{\mathrm{aq}}^{-} \leftrightarrows \underbrace{\mathrm{INDH}_{\mathrm{m}}^{+}}_{\text {COLORLESS }}+\mathrm{R}^{+} \mathrm{AN}_{\mathrm{m}}^{-}}_{\text {YELLOW }}
$$

Anion-selective with

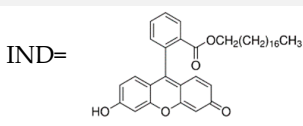

$\mathrm{R}^{+}=\left[\mathrm{CH}_{3}\left(\mathrm{CH}_{2}\right)_{11}\right]_{3} \mathrm{~N}^{+} \mathrm{CH}_{3}$

$\mathrm{AN}=$ anion, e.g., $\mathrm{ClO}_{4}^{-}$
2

Cation-selective with Chromoionophore I

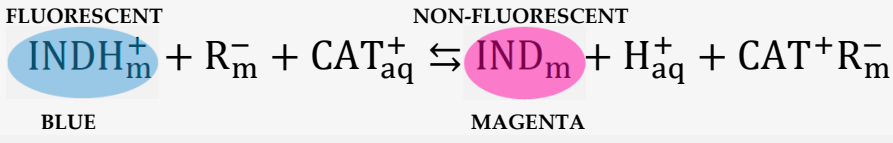

$\mathrm{IND}=$

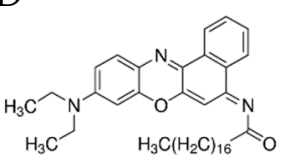

3

Chromoionophore IV
Amine-selective with

$$
\mathrm{INDH}_{\mathrm{m}}^{+}+\mathrm{RNH}_{3 \text { aq }}^{+}+\mathrm{A}
$$

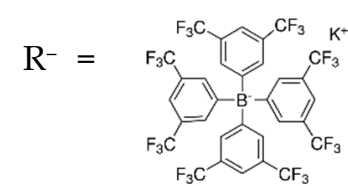

Chromoionophore IV $\mathrm{IND}=$

$\mathrm{AI}=$ amine ionophore
$\mathrm{RNH}_{3}{ }^{+}=$amine, e.g., PEA

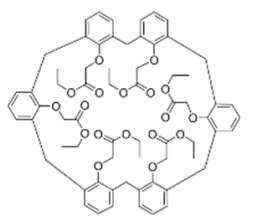

$\begin{gathered}4 \\ \text { with } \\ \text { Chromoionophore XI }\end{gathered}$
$\mathrm{IND}=$


Table 2. Calibration parameters for the developed microspheres in the presence of their model analytes (PEA ${ }^{+}$- phenethylamine, IPA—isopropyl alcohol).

\begin{tabular}{|c|c|c|c|c|c|c|c|c|c|}
\hline \multirow[b]{2}{*}{ Microsphere Type } & \multirow{2}{*}{$\begin{array}{c}\text { Model } \\
\text { Analyte }\end{array}$} & \multicolumn{4}{|c|}{ Spectrophotometric Calibration } & \multicolumn{4}{|c|}{ Spectrofluorimetric Calibration } \\
\hline & & $\lambda_{\max }$ & $\begin{array}{c}\text { Linear Range/ } \\
\log (\mathrm{c} / \mathrm{M})\end{array}$ & Slope & $\mathbf{R}^{2}$ & $\lambda_{\mathrm{ex}} / \lambda_{\mathrm{em}}$ & $\begin{array}{c}\text { Linear Range/ } \\
\log (\mathrm{c} / \mathrm{M})\end{array}$ & Slope & $\mathbf{R}^{2}$ \\
\hline $\begin{array}{c}1 \\
\text { Anion-selective with } \\
\text { Chromoionophore XI }\end{array}$ & $\mathrm{ClO}_{4}^{-}$ & $455 \mathrm{~nm}$ & $(-6.0)-(-1.0)$ & -0.016 & 0.973 & $\begin{array}{l}463 \mathrm{~nm} / \\
555 \mathrm{~nm}\end{array}$ & $(-6.0)-(-1.0)$ & 10,505 & 0.972 \\
\hline $\begin{array}{c}2 \\
\text { Cation-selective with } \\
\text { Chromoionophore I }\end{array}$ & $\mathrm{NH}_{4}^{+}$ & $612 \mathrm{~nm}$ & $(-5.5)-(-1.0)$ & -0.036 & 0.962 & $\begin{array}{l}614 \mathrm{~nm} / \\
686 \mathrm{~nm}\end{array}$ & $(-6.0)-(-1.5)$ & -540 & 0.978 \\
\hline $\begin{array}{c}\text { 3 } \\
\text { Amine-selective with } \\
\text { Chromoionophore IV }\end{array}$ & $\mathrm{PEA}^{+}$ & $450 \mathrm{~nm}$ & $(-3.0)-(-1.0)$ & 0.544 & 0.908 & - & - & - & - \\
\hline $\begin{array}{c}4 \\
\text {-OH group sensitive with } \\
\text { Chromoionophore XI }\end{array}$ & IPA & - & - & - & - & $\begin{array}{l}463 \mathrm{~nm} / \\
555 \mathrm{~nm}\end{array}$ & $(-4.0)-(-1.0)$ & 1809 & 0.870 \\
\hline
\end{tabular}

\subsection{Data Analysis}

All data were obtained in the form of absorption spectra or emission curves recorded for respective excitation wavelengths (Table 2). From the curves, point values were extracted and used for the determination of calibration characteristics; otherwise, in the case of neurotransmitters identification, whole curves served as fingerprints for specified bioanalytes. The possibility of the identification was first verified by means of unsupervised chemometric technique-Principal Component Analysis (PCA); then, Partial Least Squares-Discriminant Analysis (PLS-DA) was applied. Prior to chemometric analysis, preprocessing was applied. All data analysis was performed using Solo software (Eigenvector Research Inc., Manson, WA, USA), while calibration figures were generated in MS Excel 2020 (Microsoft, Redmond, WA, USA).

\section{Results and Discussion}

\subsection{Chemosensory Properties of the Microspheres}

Four types of microspheres were prepared according to the procedure given in the Experimental section, whose chemosensory properties were confirmed by calibration toward model analytes (Tables 1 and 2). First, cation-selective microsphere suspensions were produced-this kind of optode is based on potassium tetraphenylborate salt as an ion exchanger, facilitating the exchange of cations between the aqueous and organic phase, which should lead to the deprotonation of chromoionophore (to preserve the electroneutrality condition of the lipophilic phase), which is reported by change of its optical properties. The sensory response to model lipophilic cations, i.e., $\mathrm{NH}_{4}{ }^{+}$, was examined using spectrophotometric measurements. In each well of a microplate, $100 \mu \mathrm{L}$ solutions containing ammonium nitrate in concentrations from $1 \mu \mathrm{M}$ to $0.1 \mathrm{M}$ were mixed with $100 \mu \mathrm{L}$ of the microsphere solution. All solutions were buffered (Tris-HCl, $\mathrm{pH}$ 9.0) to make the changes of the chromoionophore protonation degree independent of $\mathrm{pH}$ to ensure that the resulting absorbance should be only influenced by differing concentrations of $\mathrm{NH}_{4}{ }^{+}$ions. The resulting UV-Vis spectra are presented in Figure 1a. Additionally, UV-Vis spectra were also recorded for the microspheres with the addition of $0.1 \mathrm{M} \mathrm{HCl}$ or $0.1 \mathrm{M} \mathrm{NaOH}$ to observe signals for fully protonated or fully deprotonated (respectively) chromoionophore. The characteristic spectrum of the protonated form of Chromoionophore I was observed with absorption maxima at $612 \mathrm{~nm}$ and $660 \mathrm{~nm}$, whereas the maximum for the deprotonated form was noted at $545 \mathrm{~nm}$, and an isosbestic point occurred at $565 \mathrm{~nm}$-all these findings are in good accordance with literature data [12,21]. The UV-Vis spectra of microspheres with $\mathrm{NH}_{4} \mathrm{NO}_{3}$ additions presented intermediate characteristics between the spectra obtained for fully protonated and deprotonated forms, indicating a partial protonation of the chromoionophore in the presence of lipophilic cations. With the increase in their concentration, the observed 
spectrum gradually becomes more similar to the spectrum obtained for a fully deprotonated chromoionophore (the degree of protonation of the chromoionophore decreases), which indicates the mechanism of proton- $\mathrm{NH}_{4}{ }^{+}$cation exchange in the lipophilic phase of the microspheres (the exchange equation for the cation-selective microspheres is shown in Table 1).

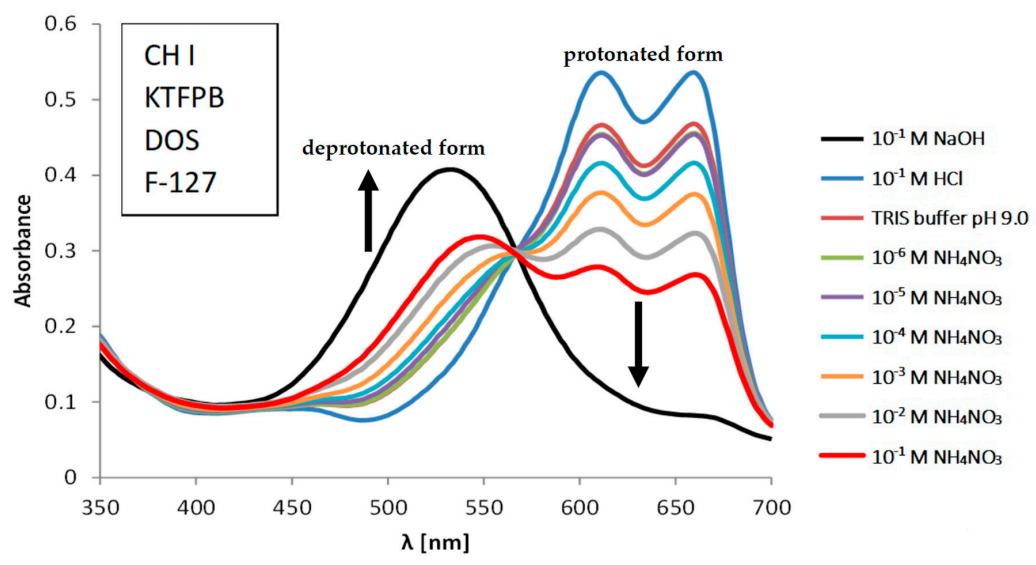

(a)

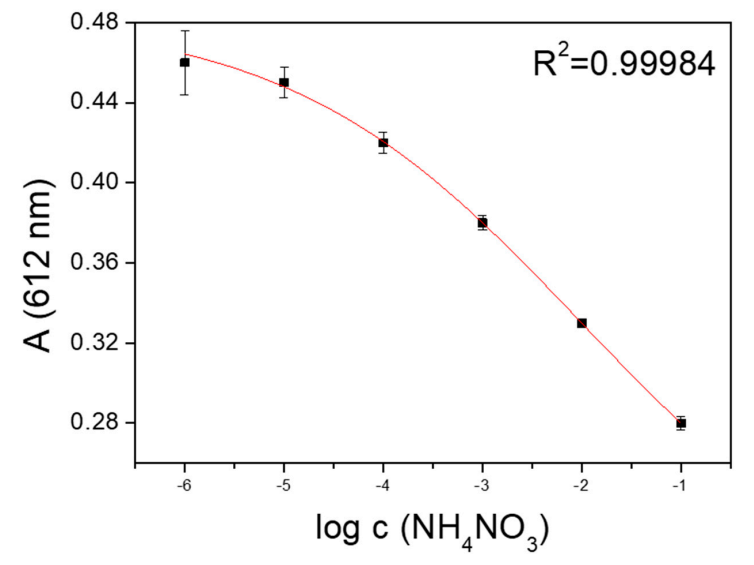

(b)

Figure 1. UV-Vis spectra (a) and calibration curve (b) for microspheres sensitive toward lipophilic cations. Each solution of $\mathrm{NH}_{4}{ }^{+}$ions was buffered (0.01 M TRIS, $\mathrm{pH}$ 9.0). Points of the calibration curve were determined as mean $\pm \mathrm{SD} ; n=4$ and were fitted with sigmoidal function.

Thus, a calibration curve was determined for cation-selective microspheres based on absorbance obtained at the maximum peak of the protonated form $(612 \mathrm{~nm})$ at varying $\mathrm{NH}_{4}{ }^{+}$concentrations (Figure $1 \mathrm{~b}$ ). Very small error bars were obtained for four replications, especially for concentrations greater than $10 \mu \mathrm{M}$, which indicates the very good repeatability of the measurements. The linear range for the obtained calibration curve is very wide-it covers the entire range of the tested concentrations, i.e., from $1 \mu \mathrm{M}$ to $0.1 \mathrm{M}$. The high value of the determination coefficient (0.96) was noticed for the linear fit of the linear range of the calibration curve. When cation-selective microspheres were examined in the fluorescence mode, an even higher determination coefficient was obtained for the linear fit of the linear range of the calibration curve (0.98), while the linear range was slightly narrower, reaching 4.5 decades (Table 2 ).

The exemplary fluorescence response of the developed microspheres is shown in an example of anion-selective micro-optodes (Figure 2). This kind of optode contains tridodecylammonium chloride as an ion exchanger (lipophilic salt), which facilitates the exchange of anions between the aqueous and organic phases. The sensory response-the fluorescence intensity of the fabricated suspension-was examined at the excitation wavelength of $463 \mathrm{~nm}$ due to the presence of chromoionophore XI ( $\lambda_{\mathrm{ex}} / \lambda_{\mathrm{em}} 463 \mathrm{~nm} / 527 \mathrm{~nm}$ [19]). The model analyte, in this case, was perchlorate anions exhibiting high lipophilicity. As before, $100 \mu \mathrm{L}$ of the analyte solution was added to an aliquot of the microsphere solution in concentrations ranging from $1 \mu \mathrm{M}$ to $0.1 \mathrm{M}$. Accordingly, all calibrating solutions were buffered (phosphate buffer $\mathrm{pH}$ 7.4) to observe the spectra changes caused only by $\mathrm{ClO}_{4}{ }^{-}$ ion. Additionally, the emission spectra for the microspheres were also recorded in the presence of $0.1 \mathrm{M} \mathrm{HCl}$ and $0.1 \mathrm{M} \mathrm{NaOH}$ to observe the signal of the fully protonated and deprotonated (respectively) form of the chromoionophore. In Figure 2a, it can be observed that the protonation of the chromoionophore causes the quenching of fluorescence (no signal in the presence of $0.1 \mathrm{M} \mathrm{HCl}$ due to the lack of fluorescent anionic form; a fully protonated chromoionophore does not exhibit fluorescence [19]), and as the $\mathrm{ClO}_{4}{ }^{-}$anions concentration increases, the fluorescence intensity decreases. It would suggest the coextraction mechanism of the sensory response-while perchlorate ions penetrate inside the microsphere, the coextraction of $\mathrm{H}^{+}$cations is forced by the electroneutrality condition; 
thus, an increase in lipophilic anions concentration leads to a higher protonation degree of chromoionophore (the exchange equation for anion-selective microspheres is shown in Table 1 [20]).

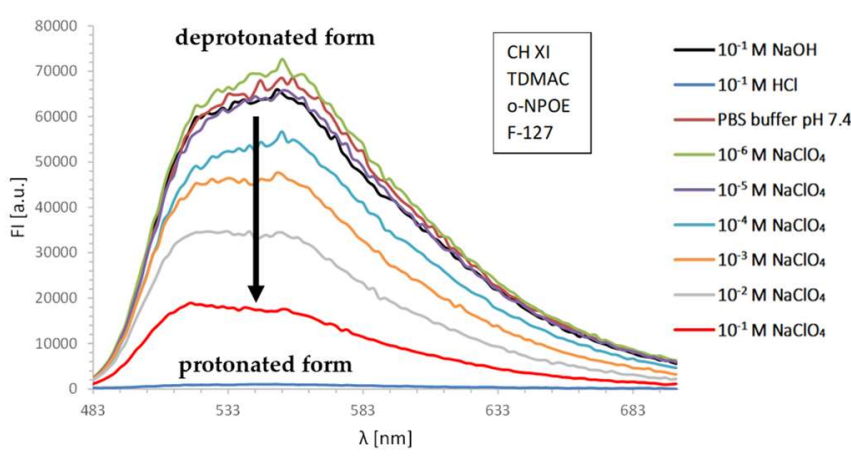

(a)

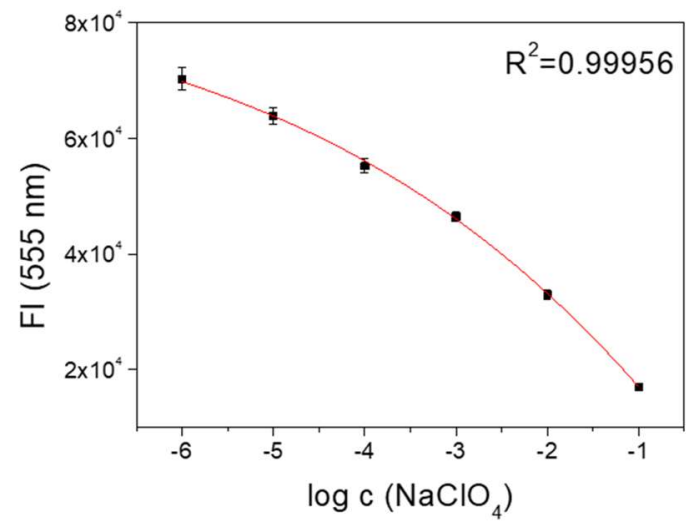

(b)

Figure 2. Fluorescence emission spectra (a) and calibration curve (b) for microspheres sensitive toward lipophilic anions. Each solution of $\mathrm{ClO}_{4}{ }^{-}$ions was buffered (0.01 M PBS, pH 7.4). Points of the calibration curve were determined as mean $\pm \mathrm{SD} ; n=4$ and were fitted with sigmoidal function.

A calibration curve was established for the anion-selective microspheres based on the fluorescence intensity recorded at $555 \mathrm{~nm}$ and presented in Figure $2 \mathrm{~b}$. The small error bars obtained for four replicates for each concentration indicate the very good repeatability of the measurement method. The linear range for the obtained calibration curve is very wide; namely, it covers the entire range of tested concentrations, i.e., from $1 \mu \mathrm{M}$ to $0.1 \mathrm{M}$. The same range as well as a high determination coefficient were obtained in the case of spectrophotometric measurements for these types of microspheres (Table 2), which confirms that both anion- and cation-selective microspheres exhibit chemosensory responses in both modes - spectrophotometric and fluorimetric. This is a very beneficial effect because they can be used as dual detection optodes, which allows increasing the accuracy and precision of the determinations.

The chemosensory properties were also confirmed for the remaining two types of microspheres-sensitive to compounds containing the amino group and sensitive to compounds containing the hydroxyl group (Tables 1 and 2). As before, calibration measurements were performed in the presence of model analytes. In the case of the amino-selective optodes, the maximum absorption peak in the UV-Vis spectra was observed at $450 \mathrm{~nm}$, which correlates well with the literature data for chromoionophore IV $[23,24]$. The change in the protonation degree of the chromoionophore is related to proton-amine cation exchange (exchange equation for amine-selective microspheres in Table 1). No fluorescence response was observed with this system. On the other hand, in the case of microspheres sensitive to compounds with the - $\mathrm{OH}$ group, a fluorescence response was observed, while changes in the UV-Vis spectra did not allow obtaining a satisfactory calibration curve. The optodes sensitive toward compounds with the -OH group exhibited the fluorescence response at excitation and emission wavelengths typical for chromoionophore XI [19]. This is the only type of microspheres studied here whose signal generation is not based on the change of protonation degree of chromoionophore. Instead, the response mechanism relies on the fact that Chromoionophore XI can reversibly recognize alcohol molecules due to the hydrogen bonding formation between Chromoionophore XI and -OH moiety, which leads to fluorescence enhancement [19] (equation for -OH group sensitive microspheres in Table 1). In comparison to the cation- and anion-selective microspheres, the amino selective and $-\mathrm{OH}$ sensitive optodes do not allow measurements in two detection modes and are also characterized by a narrower linear range of response and slightly lower determination coefficient (Table 2). 


\subsection{Time Stability and Fabrication Repeatability of the Microspheres}

The developed microspheres are colloidal systems for which the reproducibility of production and stability over time are important aspects influencing their usability because they determine the repeatability of their analytical performance. The time stability of the microspheres was studied with the use of the anion-selective microsphere system as an example (the composition, preparation, and measurement procedure is the same as in the Chemosensory properties of microspheres section). The sensory response of the system (Figure 2) was checked within 2 months. UV-Vis spectra at a varying concentrations of the model lipophilic anions as well as spectra in highly acidic and highly basic conditions were recorded in each time point (freshly prepared microspheres-START, after 1 or 2 weeks storage, after 2 months storage; see Figure 3). The obtained calibration curves were slightly biased while maintaining similar sensitivity and linear range. Thus, for a clearer comparison of chemosensory properties, the signals were expressed in the form of a protonation degree of the chromoionophore $1-\alpha$, which means the normalization based on the absorbances at peak maxima for the fully protonated and fully deprotonated form of chromoionophore [2] Then, the calibration curves were presented based on the change of the protonation degree of the chromoionophore $\Delta(1-\alpha)$ at varying perchlorate ions concentrations. It can be observed in Figure 3A that highly similar calibration curves were obtained with low values of error showing good repeatability of the measurements. It results from the high repeatability of the protonation degree of the chromoionophore for a given concentration of the model analyte in various time points. The presented results prove the very good stability of the examined microspheres within 2 months-for such a long time, it is possible to obtain reproducible results with their application.

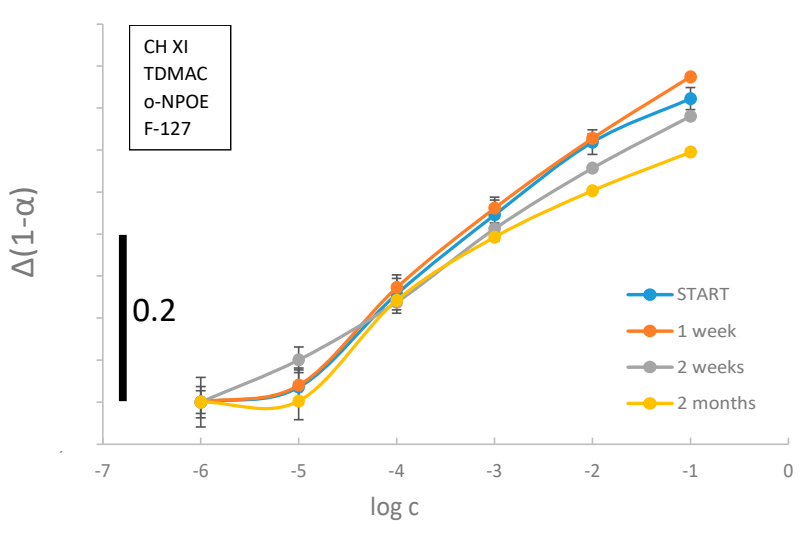

(a)

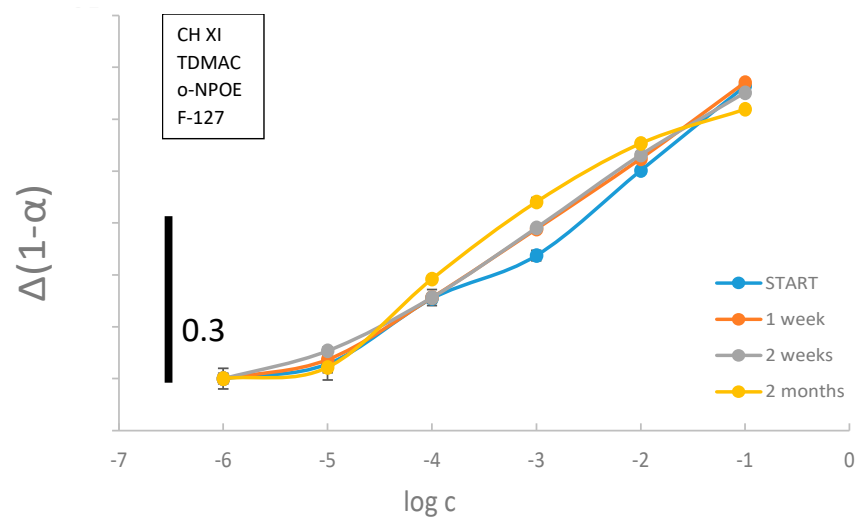

(b)

Figure 3. Time stability of chemosensory performance of microspheres sensitive toward lipophilic anions: (a) for UV-Vis spectra, (b) for fluorescence emission spectra. Points of calibration curves were determined for $\mathrm{NaClO}_{4}$ as model analyte, as mean $\pm \mathrm{SD} ; n=4$. Chemosensory responses were presented as a change in the protonation degree $(1-\alpha)$ of chromoionophore.

For the same type of microspheres, the repeatability of fluorescent sensory response was tested in a two-month period (Figure 3b). At each time point, similar changes in emission spectra were noted, as observed in Figure 2a. Again, for a clearer comparison of the chemosensory performance for various time points, the signals were expressed as the change in the protonation degree of the chromoionophore $\Delta(1-\alpha)$. In addition, the spectrofluorimetric study revealed a high temporal stability of the optode microspheres due to the high similarity of the calibration curves resulting from the high repeatability of the protonation degree of chromoionophore received, regardless of the storage time. The obtained results prove the very good stability of the fluorescence response of the examined microsphere suspensions within 2 months. For at least such a long time, it is possible to use microspheres optodes both in absorbance and fluorescence mode. 
The repeatability of fabrication is a key issue for sensor development as well as for micro- and nanostructures manufacturing. Thus, we decided to check the repeatability of chemosensory performance for the independent fabrication of lots of optode microspheres. Each of the four lots was prepared separately, independent from each other, according to the standard procedure for anion-selective microspheres used in this work. At a varying concentration of perchlorate ions, spectrophotometric UV-Vis, as well as emission spectra, were recorded for each lot, as well as the spectra of microspheres suspensions in highly acidic and highly basic conditions for each lot separately. The results were again presented based on the protonation degree of the chromoionophore (Figure 4). As can be seen from calibration curves obtained for absorbance and fluorescence measurements (Figure 4A,B, respectively), sensory characteristics are highly similar for various independently prepared lots of the developed microsphere optodes; thus, the fabrication procedure can be regarded as very repeatable.

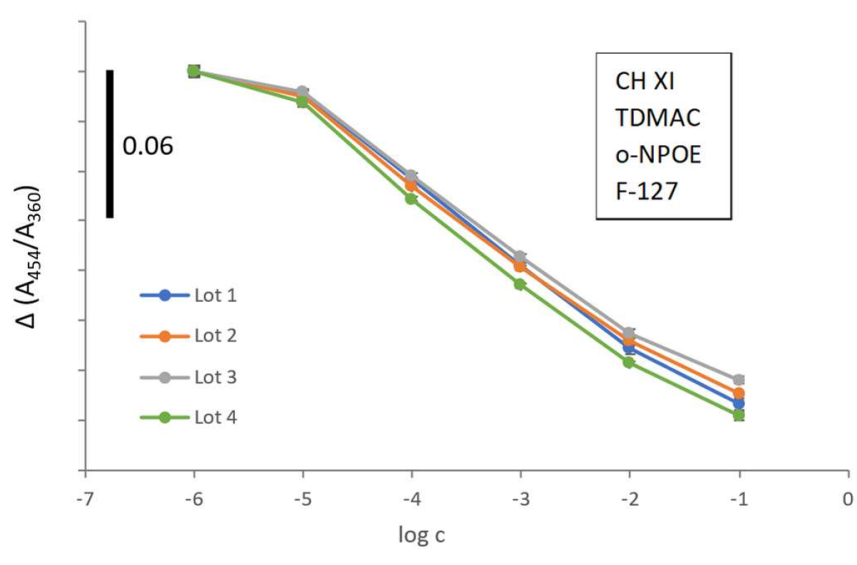

(a)

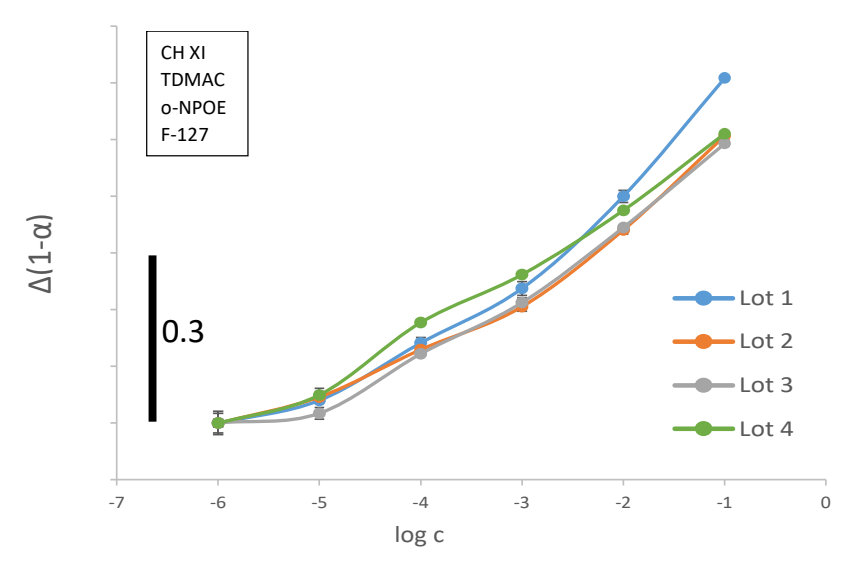

(b)

Figure 4. Repeatability of chemosensory performance for the independent fabrication of lots of microspheres sensitive toward lipophilic anions: (a) for UV-Vis spectra (b) for fluorescence emission spectra. Points of calibration curves were determined for $\mathrm{NaClO}_{4}$ as model analyte, as mean \pm SD; $n=4$. Chemosensory responses were presented as (a) change of baseline corrected absorbance (ratio of absorbances at 454 and $360 \mathrm{~nm})$ or $(\mathbf{b})$ protonation degree $(1-\alpha)$ of chromoionophore.

\subsection{Discrimination and Identification of Neurotransmitters Based on Differential Sensing}

Differential sensing techniques (also known as electronic nose/tongue or chemical nose) are becoming nowadays an attractive alternative to the classical selective/specific identification of analytes due to the possibility of using various not highly specific, having different binding affinities receptors or sensors whose response pattern is decoded by numerical processing $[1,9,10,25-27]$. This concept is based on mimicking natural chemical senses. Mammalian olfaction and gustation employ cross-reactive receptors that interact differentially with odorants and tastants. Instead of identifying an odorant or tastant molecule by its strong affinity for one particular receptor, recognition is achieved by the composite response of the array of cross-selective receptors in the nose or on the tongue. The result is a characteristic pattern-a fingerprint that can be perceived by the brain and stored in an organism's memory. Arrays of cross-sensitive receptors allow providing a characteristic fingerprint for investigated samples exactly in the same manner. The information hidden in such a fingerprint is not accessible or straightforward (via standard calibration) - it must be deconvoluted by numerical processing for the identification, recognition, classification, and/or quantification of various analytes that have a similar structure. During the last few years, there have been great achievements in this field, showing a wide applicability of differential sensing strategies, including use in medical diagnostics and drug discovery [28-32]. 
To check the possibility of using the developed microspheres as differential microsensors, their classification ability toward the identification and recognition of model analytes was studied. As a library of model compounds to be identified, eight neurotransmitters were chosen, whose similarity degree was varied. Part of them belong to catecholamines, and thus, their chemical structure is quite similar (dopamine, epinephrine, norepinephrine), while the structure of the rest is quite differentiated (GABA, acetylcholine, phenylethylamine, histamine, taurine). However, all these compounds have amino and/or hydroxyl groups and present various degrees of lipophilicity. Therefore, sensors responding to these properties shall be helpful in the recognition of this class of compounds. Microspheres optodes having sensitivity toward lipophilic cations, lipophilic anions, and compounds with amino- and $-\mathrm{OH}$ groups (i.e., types $1-4$ in Tables 1 and 2) could form a microsphere array applicable in this task.

We decided to use all four kinds of the developed microspheres, whose optical properties were tested in the presence of the eight neurotransmitters. Each kind of microsphere suspension was prepared just before use (two independent fabrication lots of each kind of microspheres), and $100 \mu \mathrm{L}$ portions were pipetted to the microwells of a microtiter plate. Then, buffered solutions of each type of neurotransmitter $(100 \mu \mathrm{M})$ were added $(1: 1 \mathrm{v} / \mathrm{v}$; eight replicates; final concentration of a neurotransmitter in a well $500 \mu \mathrm{M}$ ) after which the spectrophotometric and/or spectrofluorimetric response of the optodes was recorded. At the first phase of the experiment, point values of absorbances at peak maximum and/or fluorescence intensities at peak maxima (for the respective excitation wavelength for each chromoionophore used, see Table 2) were applied. Thus, the data matrix of size 64 samples $\times 6$ features was processed by Principal Component Analysis (PCA), and the resulting score plot is presented in Figure 5.

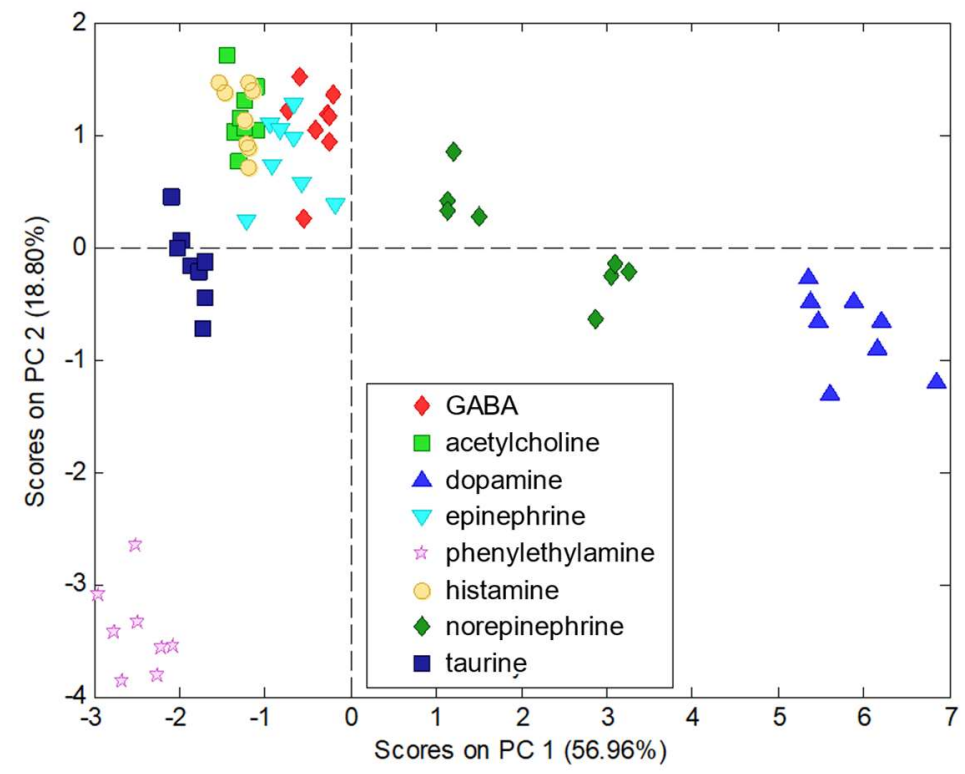

Figure 5. PCA score plot showing the identification of neurotransmitters by means of the studied set of chemosensory microspheres. As original features, point values of absorbances@peak maxima and/or fluorescence intensities@peak maxima (for respective excitation wavelength for each chromoionophore used) were applied. For preprocessing, only autoscaling was used.

PCA is an unsupervised data analysis technique used usually for the reduction of original data to the most informative ones, having the highest ability for discrimination of the objects characterized by multidimensional features. In this case, the first two PCs contained over $75 \%$ of the variance of the dataset, which means that there is still $25 \%$ of variance not included in the PCA score plot. There are clusters that are clearly separable, such as norepinephrine, dopamine, phenylethylamine, and taurine clusters, while the remaining four are partially overlapping. We applied PCA on point data without any 
additional preprocessing except autoscaling as the most straightforward technique to prove the discrimination capability of the studied optode set. We can distinguish three groups of samples on the PCA score plot: catecholamines having both amino and -OH groups, phenylethylamine with an amine group and without an - $\mathrm{OH}$ group, and the rest of the neurotransmitters, which also have an amine group and lack an -OH group, but they are far less lipophilic than phenylethylamine $(\log p=1.41$, the rest of the compounds have $\log p<0)$. Thus, $\mathrm{PC} 1$ can be related to the presence of the $-\mathrm{OH}$ group-compounds without this moiety in the molecule exhibit PC1 $<0$, whereas higher values of PC1 were observed for norepinephrine and dopamine (two-OH groups). It is in accordance with PC1 loadings - the highest contribution to this loading had a fluorescence signal of microspheres detecting the -OH group. On the other hand, PC2 can discriminate lipophilicity; the lowest value of PC2 was observed for phenylethylamine. Moreover, looking at the catecholamines, a systematic decrease in PC2 score correlates with $\log p(-1.37,-1.24$, -0.98 for epinephrine, norepinephrine, and dopamine, respectively). The highest PC2 loading was observed for a fluorescence signal of cation-selective microspheres having affinity toward lipophilic cations, including amines, which explains the discriminative ability of PC2 in terms of lipophilicity.

We decided to use whole spectra of spectrophotometric and spectrofluorimetric responses (instead of point values) for the enhanced discrimination of the neurotransmitters. Moreover, the supervised data analysis technique could be helpful to more effectively find discriminative features in such an enlarged data matrix; therefore, we applied Partial Least Squares-Discriminant Analysis (PLS-DA) in the second phase of the differential sensing experiment. As was supposed, the use of the supervised method allowed obtaining satisfactory discrimination of the samples, which is visible in the PLS-DA score plot given in Figure 6. All clusters are clearly visible; the only small overlapping occurs for histamine and acetylcholine samples. As $68 \%$ of the variance is observed in this plot, the other $32 \%$ could give a subtle differentiation between the two problematic samples. It is worth noticing that the grouping of catecholamines (epinephrine, norepinephrine, dopamine) in one supercluster can be observed and phenylethylamine having the same amino-ethylphenyl moiety is also significantly distinct from the other four samples.

On the other hand, one can observe the substructure of clusters of catecholamines and phenylethylamine, which are marked with a dotted line in Figure 6. This effect is linked with the fact that two independent lots of each kind of microsphere were applied for this study. As it was shown above (Figure 4), their responses are highly similar but not identical, which is also reflected in the PLS-DA plot of neurotransmitters. Nevertheless, even though the two lots give a slightly different placement of clusters for the same compound, still, the location on the PLS-DA plot is characteristic and is another form of evidence for the satisfactory fabrication repeatability of the developed microspheres. However, this effect should be verified in another experiment, in which discrimination of the neurotransmitters was achieved with one lot of the microspheres, whereas the recognition was based on signals obtained for another: the independent fabrication lot of the micro-optodes. Thus, the data (64 samples, eight neurotransmitters in eight replicates) were split into two subsets-the train set formed by the signals of the first fabrication lot of each kind of microspheres (32 samples $=8$ neurotransmitters in 4 replicates), and the test set, with signals recorded for the second, independent fabrication lot (32 samples = eight neurotransmitters in four replicates). The PLS-DA model was established based on the train set, and its generalized recognition capability was verified by the independent, external test set. For each of the 32 samples, the most probable class was determined. Great accuracy $(100 \%$ for both the train and test set) was obtained; all samples were perfectly recognized with the use of the developed microsphere array. It again shows good repeatability of chemosensory properties of the optodes as well as the great ability of such microparticles to be used in differential sensing. 


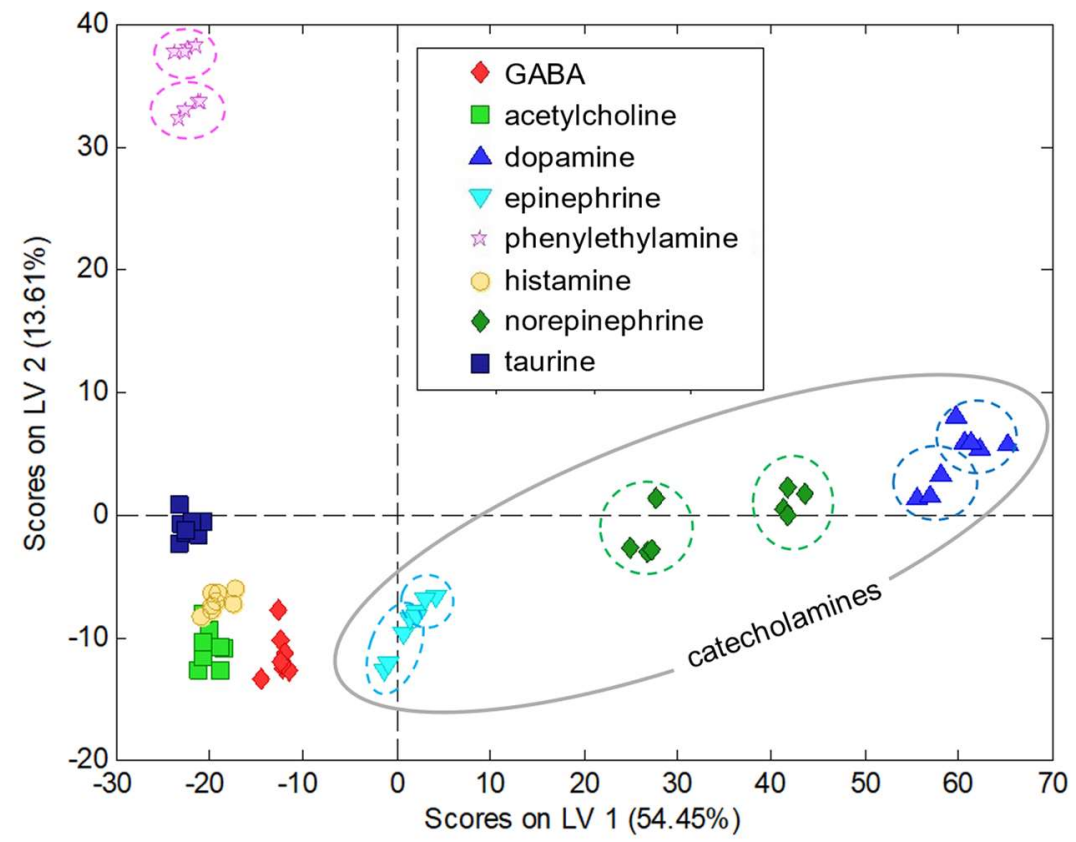

Figure 6. PLS-DA score plot showing the identification of neurotransmitters by means of the studied set of chemosensory microspheres. As original features, whole response curves (UV-Vis spectra and/or emission spectra for respective excitation wavelength for each chromoionophore used) were applied. For preprocessing, Savitzky-Golay smoothing and differentiation was used. Color ovals represent the identification of neurotransmitters obtained with two independent fabrication lots of microspheres-this discrimination is more visible for catecholamines and phenylehylamine.

3.4. Quantification of Neurotransmitters and Selectivity of Chemosensory Microsphere Set in a Blood Plasma Solution

The following step was to check if the developed microsphere set can discern various levels of concentration of the studied neurotransmitters and if the quantification can be performed in mixtures. We prepared 45 samples differing in composition, containing dopamine, histamine, and phenylethylamine in micromolar to millimolar concentration (composition of samples given in Figure 7a). Part of the samples contained only dopamine at various concentration levels (samples 1-20), part of them contained only histamine at various concentration levels (samples 21-35), and the last 10 samples were mixtures of neurotransmitters.

PLS analysis of micro-optodes array signals revealed the possibility of quantifying the studied neurotransmitters (Figure 7). Dopamine concentration was predicted with satisfactory accuracy for concentration levels ranging from $1 \mu \mathrm{M}$ to $1 \mathrm{mM}$ (samples 1-20, Figure $7 \mathrm{~b}$ ), also when determined in the mixture with phenylethylamine (samples 36-40, Figure 7b). In samples of histamine on various concentration levels as well as in the mixture of histamine and phenylethylamine, this analyte level was predicted as "not observed" (samples 21-35 and 41-45, Figure 7b). Non-catecholamine neurotransmitter histamine could have been determined in concentration levels from $10 \mu \mathrm{M}$ to $1 \mathrm{mM}$ (samples 21-35, Figure 7c), and its determination in the presence of phenylethylamine (samples 41-45, Figure 7c) also provided a satisfactory result. In the case of samples 1-20 and 36-40 that contained various concentrations of dopamine or a mixture of dopamine and phenylethylamine, PLS prediction gave the correct output: "not observed" (Figure 7c). Phenylethylamine was present only in samples 36-45, but not as a single analyte-it was in mixtures with dopamine or histamine. Even though the concentration was correctly predicted both for these mixtures (samples 36-45, Figure 7d) and for samples not containing this analyte (samples 1-35, Figure 7d), these results clearly show the possibility of quantification of the studied compounds even when they are present in mixtures. 
(a)

\begin{tabular}{|c|c|c|c|c|c|}
\hline \multicolumn{2}{|c|}{ samples } & \multicolumn{3}{|c|}{ concentration/log c } & \\
\hline & & DA & HIS & PEA & \\
\hline $1-5$ & $2 \pi$ & -3 & - & - & \\
\hline 6-10 & $\nabla$ & -4 & - & - & -only DA \\
\hline $11-15$ & $\Delta$ & -5 & - & - & \\
\hline $16-20$ & $\square$ & -6 & - & - & \\
\hline $21-25$ & $\Delta$ & - & -3 & - & \\
\hline $26-30$ & $\square$ & - & -4 & - & only HIS \\
\hline $31-35$ & 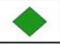 & - & -5 & - & \\
\hline $36-40$ & $\bullet$ & -3 & - & -3 & $D A+P E A$ \\
\hline $41-45$ & 0 & - & -3 & -3 & HIS+PEA \\
\hline
\end{tabular}

(c)

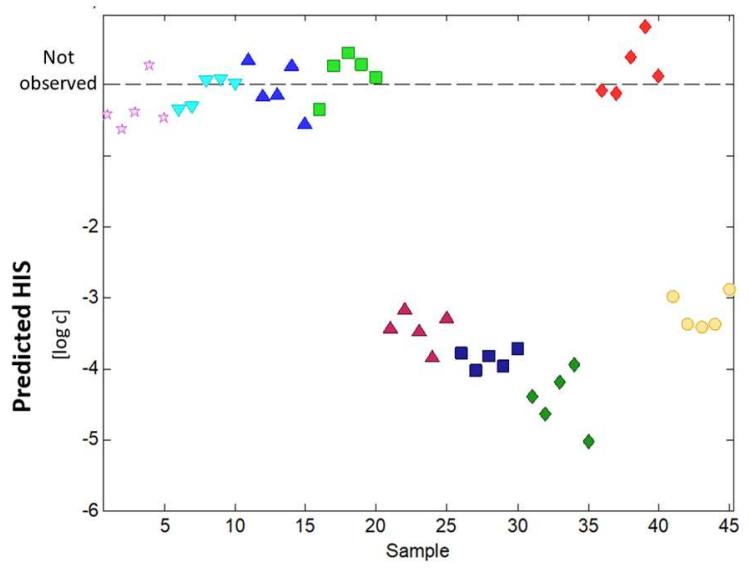

(b)

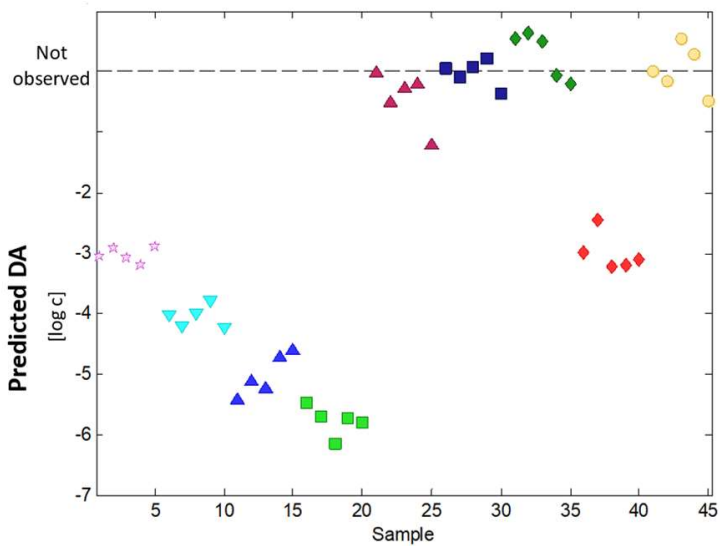

(d)

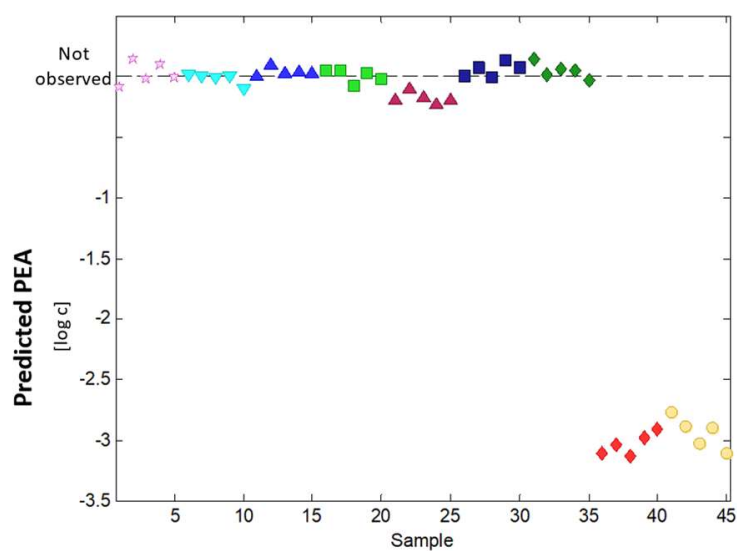

Figure 7. PLS prediction of the concentration level of dopamine (DA), histamine (HIS), and phenylethylamine (PEA) in 45 samples: (a) composition of the samples; (b) PLS-predicted DA level; (c) PLS-predicted HIS level; (d) PLS-predicted PEA level.

Various biomolecules having similar functional groups could interfere in the analysis performed with the developed microspheres, especially in biological media. Thus, we studied the selectivity of the microsphere array on an example of amino acids in simulated blood plasma solution (BPS). For this study, we used samples of three neurotransmitters (dopamine, norepinephrine, histamine), four proteinogenic amino acids (Ala with aliphatic side-chain, Asp with amide side-chain, Pro with cyclized side-chain, Tyr with aromatic side-chain), and taurine, which is both an amino acid and neurotransmitter. As shown in Figure 8a, catecholamine neurotransmitters (dopamine and norepinephrine) and noncatecholamine histamine are easily discernable from the studied amino acids Ala, Asp, Pro Tyr, and taurine (all in BPS). Their clusters are an insignificant distance from pure BPS compared to all amino acids having a more similar signal pattern to pure BPS. This indicates the significant influence of neurotransmitters on microspheres' signals and the much-limited impact of amino acids, which in turn shows the superior selectivity of the developed micro-optode set toward the neurotransmitters. 
(a)

(b)

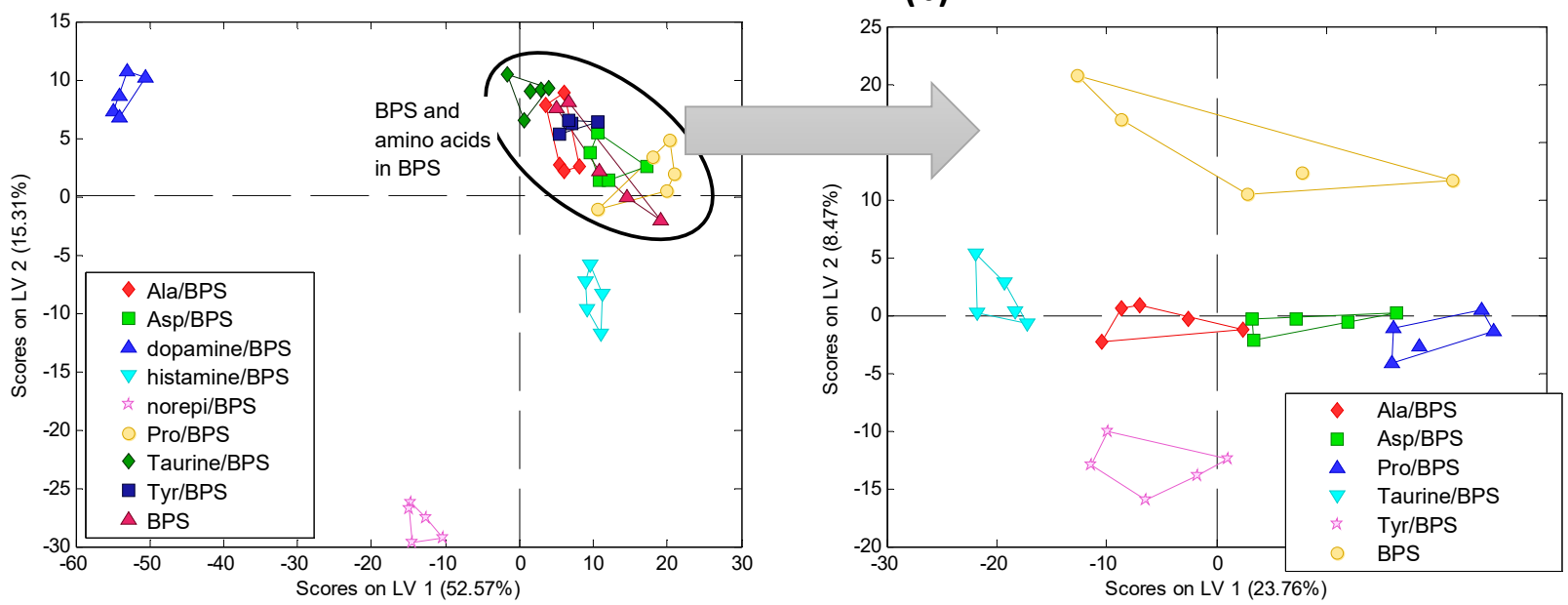

Figure 8. PLS-DA score plots show the discrimination of neurotransmitters and amino acids in artificial blood plasma solution (BPS) using the studied set of chemosensory microspheres. As original features, whole response curves (UV-Vis spectra and/or emission spectra for respective excitation wavelength for each chromoionophore used) were applied. For preprocessing, SavitzkyGolay smoothing and differentiation were used. Samples indicated in the black oval in (a) were subjected to independent PLS-DA and are shown in (b).

Samples belonging to the cluster marked with an oval in Figure 8a were studied further to investigate if they can be differentiated from pure BPS. The result of PLS-DA is shown in Figure 8b. The presence of all amino acids was detected-clusters of amino acids in BPS are discernable from pure BPS. Moreover, clusters of various amino acids are also separable, which suggest a slight discrimination capability of the microsphere array also toward these bioanalytes. However, it is much smaller, which is evidenced by the smaller distances between the clusters. It is worth underlining that the pattern of clusters reflects variability in the chemical structures of the studied amino acids. Aliphatic $\alpha$-amino acids (Ala, Asp, and Pro) are close to each other, forming super-clusters discernable easily from the aromatic $\alpha$-amino acid Tyr and $\beta$-amino acid taurine, with a sulfonic group instead of a carboxylic one present in the rest of the amino acids.

In contrast to amino acids, neurotransmitter compounds are not structurally similar and mainly do not have similar moiety; it is very distinctive that the only amino acid neurotransmitter applied in this work, i.e., taurine, exhibited behavior similar to other amino acids. It confirms that the developed micro-optode array has a high potential to recognize biomolecules based on their chemical moieties, toward which the fabricated microspheres were designed to be sensitive.

\section{Conclusions}

Differential sensing is a robust and effective tool in the field of identification of various analytes, even those having very similar chemical structures. It has attracted much attention during the last few years; therefore, there is a great need to develop receptors and sensors that can be applied in the form of versatile receptor/sensor arrays dedicated to the identification and quantification of analytes. In this work, we propose newly developed chemosensory microspheres based on a Pluronic surfactant as elements of an optode array used for differential sensing. As it was shown, the fabrication of these micro-optodes can be performed very repeatably, and the obtained microspheres maintain their chemosensory properties for at least 2 months. They exhibit a wide range of linear responses (even covering five orders of magnitude). Their usability as cross-sensitive elements of a sensor array was shown in an example of identification of eight neurotransmitters resulting in the perfect recognition of these bioanalytes. It was shown that the microspheres can respond in 
a dual mode of detection, spectrophotometric and fluorimetric, which can help enhance the identification accuracy. Moreover, it is possible to use the micro-optodes set in quantitative analysis, even in mixtures of analytes. It was also proved that possibly interfering amino acids are easily discernable from the studied neurotransmitters in blood plasma solution, which shows the selective behavior of the studied system. It can recognize subtle differences in chemical structure based on the differential interaction of microspheres with various moieties present in the molecule.

However, it must be underlined that this work shows only the proof of principle that the developed surfactant stabilized cross-sensitive micro-optodes can be applied for differential sensing. For a detailed picture of the performance of the proposed sensing strategy, the following research must be undertaken. As the most important ones, we consider the detailed quantitative analysis of selected bioanalytes, including analytical performance characterization in terms of LOD and LOQ, with special emphasis given to the extension of the method to nanomolar concentration range, which is demanded for wider practical usability. Further works on expanding the potential of the newly developed cross-sensitive ion-sensing microspheres are ongoing in our laboratory to explore various formulations of the optodes and their usability in the differential sensing of various biomolecules.

Author Contributions: Conceptualization, resources, writing-review and editing, supervision, project administration, funding acquisition, P.C.-S.; methodology, formal analysis, writing-original draft preparation, visualization, P.C.-S. and A.K.; validation, A.K.; investigation, data curation, A.K., P.M., M.W. All authors have read and agreed to the published version of the manuscript.

Funding: This work was financially supported by National Science Centre (Poland) within the framework of the SONATA BIS project No. UMO-2018/30/E/ST4/00481. Aleksandra Kalinowska acknowledges financial support from IDUB project (Scholarship Plus programme).

Institutional Review Board Statement: Not applicable.

Informed Consent Statement: Not applicable.

Data Availability Statement: Not applicable.

Acknowledgments: Aleksandra Kalinowska acknowledges financial support from IDUB project (Scholarship Plus programme).

Conflicts of Interest: The authors declare no conflict of interest.

\section{References}

1. You, L.; Zha, D.; Anslyn, E.V. Recent Advances in Supramolecular Analytical Chemistry Using Optical Sensing. Chem. Rev. 2015, 115, 7840-7892. [CrossRef] [PubMed]

2. Johnson, R.D.; Bachas, L.G. Ionophore-based ion-selective potentiometric and optical sensors. Anal. Bioanal. Chem. 2003, 376, 328-341. [CrossRef]

3. Ferris, M.S.; Katageri, A.G.; Gohring, G.M.; Cash, K.J. A dual-indicator strategy for controlling the response of ionophore-based optical nanosensors. Sens. Actuators B 2018, 256, 674-681. [CrossRef]

4. Bakker, E.; Bühlmann, P.; Pretsch, E. Carrier-Based Ion-Selective Electrodes and Bulk Optodes. 1. General Characteristics. Chem. Rev. 1997, 97, 3083-3132. [CrossRef]

5. Xie, X.; Bakker, E. Ion selective optodes: From the bulk to the nanoscale. Anal. Bioanal. Chem. 2015, 407, 3899-3910. [CrossRef] [PubMed]

6. Du, X.; Xie, X. Ion-Selective optodes: Alternative approaches for simplified fabrication and signaling. Sens. Actuators B Chem. 2021, 335, 129368. [CrossRef]

7. Xie, X.; Mistlberger, G.; Bakker, E. Ultrasmall Fluorescent Ion-Exchanging Nanospheres Containing Selective Ionophores. Anal. Chem. 2013, 85, 9932-9938. [CrossRef]

8. Xie, X.; Zhai, J.; Crespo, G.A.; Bakker, E. Ionophore-Based Ion-Selective Optical NanoSensors Operating in Exhaustive Sensing Mode. Anal. Chem. 2014, 86, 8770-8775. [CrossRef] [PubMed]

9. Li, Z.; Askim, J.R.; Suslick, K.S. The Optoelectronic Nose: Colorimetric and Fluorometric Sensor Arrays. Chem. Rev. 2019, 119, 231-292. [CrossRef]

10. Wong, S.; Khor, S.M. State-of-the-art of differential sensing techniques in analytical sciences. Trends Anal. Chem. 2019, 114, 108-125. [CrossRef] 
11. Przondziono, J.; Walke, W.; Hadasik, E.; Mlynarski, R. Forecasting of Corrosion Properties of Steel Wires for Production of Guide Wires for Cardiological Treatment. Adv. Mater. Sci. Eng. 2013, 2013, 1-6. [CrossRef]

12. Chan, W.H.; Lee, A.W.M.; Wang, K. Design of a primary amine-selective optode membrane based on a lipophilic hexaester of calix[6]arene. Analyst 1994, 119, 2809-2812. [CrossRef]

13. Mohr, G.J.; Citterio, D.; Demuth, C.; Fehlmann, M.; Jenny, L.; Lohse, C.; Moradian, A.; Nezel, T.; Rothmaier, M.; Spichiger, U.E. Reversible chemical reactions as the basis for optical sensors used to detect amines, alcohols and humidity. J. Mater. Chem. 1999, 9, 2259-2264. [CrossRef]

14. Wesoły, M.; Zabadaj, M.; Amelian, A.; Winnicka, K.; Wróblewski, W.; Ciosek, P. Tasting cetirizine-based microspheres with an electronic tongue. Sens. Actuators B Chem. 2017, 238, 1190-1198. [CrossRef]

15. Kutyła-Olesiuk, A.; Ciosek, P.; Romanowska, E.; Wróblewski, W. Effect of lead accumulation in maize leaves on their chemical images created by a flow-through electronic tongue. Talanta 2013, 103, 179-185. [CrossRef]

16. Jańczyk, M.; Kutyła, A.; Sollohub, K.; Wosicka, H.; Cal, K.; Ciosek, P. Electronic tongue for the detection of taste-masking microencapsulation of active pharmaceutical substances. Bioelectrochemistry 2010, 80, 94-98. [CrossRef]

17. Kalinowska, A.; Matusiak, P.A.; Skorupska, S.; Grabowska-Jadach, I.; Ciosek-Skibińska, P. Influence of the type and amount of plasticizer on the sensory properties of microspheres sensitive to lipophilic ions. In Proceedings of the CSAC2021: 1st International Electronic Conference on Chemical Sensors and Analytical Chemistry, Online, 1-15 July 2021; MDPI: Basel, Switzerland. Available online: https:/ / sciforum.net/paper/view/10487 (accessed on 17 December 2021). [CrossRef]

18. Kalinowska, A.; Skorupska, S.; Grabowska-Jadach, I.; Ciosek-Skibińska, P. The Use of Ultrasounds in the Preparation of Chemosensory Microstructures. Eng. Proc. 2021, 10, 11. [CrossRef]

19. Zeng, H.H.; Wang, K.M.; Li, D.; Yu, R.Q. Development of an alcohol optode membrane based on fluorescence enhancement of fluorescein derivatives. Talanta 1994, 41, 969-975. [CrossRef]

20. Ortuño, J.A.; Albero, M.I.; García, M.S.; Sánchez-Pedreño, C.; García, M.I.; Expósito, R. Flow-through bulk optode for the fluorimetric determination of perchlorate. Talanta 2003, 60, 563-569. [CrossRef]

21. Chan, W.H.; Lee, A.W.M.; Lee, C.M.; Yau, K.W.; Wang, K. Design and characterization of sodium-selective optode membranes based on the lipophilic tetraester of calix[4]arene. Analyst 1995, 120, 1963-1967. [CrossRef]

22. Du, X.; Zhu, C.; Xie, X. Thermochromic Ion-Exchange Micelles Containing H+ Chromoionophores. Langmuir 2017, 33, 5910-5914. [CrossRef]

23. Ambrose, T.M.; Meyerhoff, M.E. Optical ion sensing with immobilized thin films of photocrosslinked decyl methacrylate. Anal Chim. Acta 1999, 378, 119-126. [CrossRef]

24. Kim, S.B.; Kang, T.Y.; Cha, G.S.; Nam, H. Quantitative determination of heparin levels in serum with microtiter plate-format optode. Anal. Chim. Acta 2006, 557, 117-122. [CrossRef]

25. Diehl, K.L.; Anslyn, E.V. Array sensing using optical methods for detection of chemical and biological hazards. Chem. Soc. Rev. 2013, 42, 8596. [CrossRef]

26. Anzenbacher, P., Jr.; Lubal, P.; Bucek, P.; Palaciosa, M.A.; Kozelkova, M.E. A practical approach to optical cross-reactive sensor arrays. Chem. Soc. Rev. 2010, 39, 3954-3979. [CrossRef]

27. Christodoulides, N.; McRae, M.P.; Simmons, G.W.; Modak, S.S.; McDevitt, T.J. Sensors that Learn: The Evolution from Taste Fingerprints to Patterns of Early Disease Detection. Micromachines 2019, 10, 251. [CrossRef]

28. Geng, Y.; Peveler, W.J.; Rotello, V.M. Array-based "Chemical Nose" Sensing in Diagnostics and Drug Discovery. Angew. Chem. Int. Ed. 2019, 58, 5190-5200. [CrossRef]

29. Peveler, W.J.; Landis, R.F.; Yazdani, M.; Day, J.W.; Modi, R.; Carmalt, C.J.; Rosenberg, W.M.; Rotello, V.M. A Rapid and Robust Diagnostic for Liver Fibrosis Using a Multichannel Polymer Sensor Array. Adv. Mater. 2018, 30, 1800634. [CrossRef]

30. Ngernpimai, S.; Geng, Y.; Makabenta, J.M.; Landis, R.F.; Keshri, P.; Gupta, A.; Li, C.H.; Chompoosor, A.; Rotello, V.M. Rapid Identification of Biofilms Using a Robust Multichannel Polymer Sensor Array. ACS Appl. Mater. Interfaces 2019, 11, 11202-11208. [CrossRef]

31. Caracciolo, G.; Safavi-Sohi, R.; Malekzadeh, R.; Poustchi, H.; Vasighi, M.; Chiozzi, R.Z.; Capriotti, A.L.; Lagana, A.; Hajipour, M.; di Domenico, M.; et al. Disease-specific protein corona sensor arrays may have disease detection capacity. Nanoscale Horiz. 2019, 4, 1063. [CrossRef]

32. Gade, A.M.; Meadows, M.K.; Ellington, A.D.; Anslyn, E.V. Differential array sensing for cancer cell classification and novelty detection. Org. Biomol. Chem. 2017, 15, 9866. [CrossRef] [PubMed] 